\title{
Evidência de Validade de Construto do NEUPSILIN Utilizando Análise Fatorial Confirmatória
}

\author{
Evidences of Construct Validity of the NEUPSILIN \\ Using Confirmatory Factorial Analysis
}

\author{
Josiane Pawlowski ${ }^{1}$ \\ Universidade Federal do Rio de Janeiro, Brasil \\ Maria Alice de Mattos Pimenta Parente ${ }^{4}$ \\ Universidade Federal do ABC, Brasil
}

\author{
Eduardo Remor ${ }^{2}$ \\ Universidad Autónoma de Madrid, España \\ Rochele Paz Fonseca ${ }^{5}$ \\ Pontificia Universidade Católica do Rio Grande do Sul, Brasil
}

\author{
Jesusa Fumagalli de Salles ${ }^{3}$ \\ Denise Ruschel Bandeira ${ }^{6}$ \\ Universidade Federal do Rio Grande do Sul, Brasil
}

\begin{abstract}
Resumo. Objetivou-se apresentar evidências de validade de construto do Instrumento de Avaliação Neuropsicológica Breve NEUPSILIN. Foram avaliados 1017 brasileiros saudáveis, 67.1\% mulheres, de 12 a 90 anos de idade $(M=36.72 ; D P$ $=23.90)$ e de 1 a 27 anos de escolaridade $(M=8.71, D P=4.13)$. Foram aplicadas as 32 tarefas do NEUPSILIN, que avaliam orientação, atenção, percepção, memória, habilidades aritméticas, linguagem, praxias e funções executivas. Modelos teóricohipotéticos, ajustados e alternativos de linguagem, memória, praxias e funções executivas foram analisados com o método de Mínimos Quadrados Generalizados do AMOS (18.0). Os modelos ajustados de linguagem e praxias, o modelo teóricohipotético de memória verbal e o modelo alternativo de funções executivas apresentaram melhores indicadores de ajuste. Os resultados apontam evidências de validade das medidas de linguagem, memória, praxias e funções executivas do NEUPSILIN.
\end{abstract}

Palavras-chave. Validade, avaliação neuropsicológica, análise fatorial.

Abstract. The objective was to provide evidences of construct validity of the NEUPSILIN Brief Neuropsychological Assessment Instrument. The participants were 1017 healthy Brazilian, $67.1 \%$ women, 12 to 90 years old $(M=36.72 ; S D=23.90)$ and 1-27 years of education $(M=8.68 ; S D=4.02)$. Participants answered the 32 tasks of NEUPSILIN that assess orientation, attention, perception, memory, arithmetic abilities, language, praxis and executive functions. Theoretical-hypothetical, adjusted and alternative models of language, memory, praxis and executive functions were analyzed with Generalized Least Squares method of AMOS (18.0). The adjusted models of language and praxis, the hypothetical-theoretical model of verbal memory and the alternative model of executive functions showed better adjustment indicators than other models. Results indicate the validity of measures of language, memory, praxis and executive functions of NEUPSILIN.

Keywords. Test validity, neuropsychological assessment, factor analysis.

\footnotetext{
${ }^{1}$ Josiane Pawlowski, Instituto de Psicologia, Universidade Federal do Rio de Janeiro, Brasil. Dirección postal: Departamento de Psicometria, Instituto de Psicologia, Pavilhão Nilton Campos, Av. Pasteur, 250, Praia Vermelha, CEP 22290-250 - Rio de Janeiro - RJ, Brasil. E-mail: josipski@gmail.com

${ }^{2}$ Eduardo Remor, Facultad de Psicología, Universidad Autónoma de Madrid, España. E-mail: eduardo.remor@uam.es

${ }_{3}^{3}$ Jerusa Fumagalli de Salles, Instituto de Psicologia e Programa de Pós-Graduação em Psicologia, Universidade Federal do Rio Grande do Sul, Brasil. E-mail: jerusafs@yahoo.com.br

${ }^{4}$ Maria Alice de Mattos Pimenta Parente, Universidade Federal do ABC, São Paulo, Brasil. E-mail: mariaalicem.pimenta@gmail.com

${ }^{5}$ Rochele Paz Fonseca, Faculdade de Psicologia e Programa de Pós-Graduação em Psicologia, Pontifícia Universidade Católica do Rio Grande do Sul, Brasil. E-mail: rochele.fonseca@gmail.com

${ }^{6}$ Denise Ruschel Bandeira, Instituto de Psicologia e Programa de Pós-Graduação em Psicologia, Universidade Federal do Rio Grande do Sul, Brasil. E-mail: deniserbandeira@gmail.com
} 


\section{Introdução}

A avaliação neuropsicológica, como um método de examinar a expressão comportamental das disfunções cerebrais, pode ser realizada mediante a aplicação no paciente de instrumentos de avaliação cognitiva (Lezak, Howieson, \& Loring, 2004; Strauss, Sherman, \& Spreen, 2006). Para a condução de uma avaliação adequada, os instrumentos de medida necessitam ter sido construídos com rigor metodológico e submetidos a estudos psicométricos que objetivam evidenciar a validade do instrumento (Pasquali, 2010; Urbina, 2004).

Segundo os Padrões de Testagem Educacional e Psicológica, publicados em 1999 pela Associação Americana de Pesquisa em Educação (AERA [American Educational Research Association]), em conjunto com a Associação Americana de Psicologia (APA [American Psychological Association]) e o Conselho Nacional de Mensurações em Educação (NCME [National Council on Measurement in Education]), a validade é entendida como o grau em que todas as evidências acumuladas apóiam a interpretação planejada dos escores de um teste para o propósito a que se destina (AERA, APA, \& NCME, 1999). Seguindo essa definição, os diferentes métodos de validação contribuem, em conjunto, para fornecer fontes de evidência de validade do novo instrumento construído.

Urbina (2004) distribui as fontes de evidência de validade em três grupos: a) Evidências de validade a partir do conteúdo do teste e de processos de resposta; b) Evidências de validade com base na exploração de padrões de convergência e divergência; e c) Evidências de validade com base em relações entre as pontuações do teste e critérios. Divergindo da visão tripartite para validação de um instrumento (validade de critério, conteúdo e construto), essa organização, que segue as regulamentações dos Padrões de Testagem Educacional e Psicológica, aponta que a soma de diferentes evidências de validade fornecem a validação de construto do novo instrumento.

Desenvolvido no Brasil, o Instrumento de Avaliação Neuropsicológica Breve NEUPSILIN (Fonseca, Salles, \& Parente, 2009) já foi submetido a diferentes estudos de evidências de validade. Esse instrumento tem o objetivo de apresentar um perfil inicial de desempenho neuropsicológico de pacientes nas habilidades cognitivas de orientação têmporoespacial, atenção, percepção, memória, aritmética, linguagem, praxias e funções executivas (resolução de problemas simples e fluência verbal). A construção do NEUPSILIN aportou-se em conceitos teóricos da Neuropsicologia, na experiência clínica de suas autoras e em outros instrumentos de medida. Esses três pilares fundamentaram a validade de conteúdo do NEUPSILIN, conforme detalhada em Fonseca, Salles e Parente (2008), resultando na composição de oito funções cognitivas avaliadas mediante 32 tarefas, conforme apresentado no Apêndice e estudos prévios (Fonseca et al., 2008; Pawlowski, Fonseca, Salles, Parente, \& Bandeira, 2008).

Além da validação referida ao conteúdo, também foram realizados estudos que avaliaram evidências de validade de construto por padrões de convergência e divergência (Urbina, 2004) a partir de: (a) diferenças de pontuações do NEUPSILIN em consonância com as diferenças esperadas em razão da variável 'anos de estudo' (Pawlowski et al., 2008); (b) correlação dos resultados do NEUPSILIN aos instrumentos: 1) Matrizes Progressivas de Raven (Raven, Court, \& Raven, 1992), traduzido e adaptado para o Brasil por Campos (2003), em estudo de Pawlowski et al. (2008), e 2) Teste de Atenção Concentrada - AC (Cambraia, 2003), Teste de Atenção TECON-1 (Tonglet, 2003), Escala de Inteligência Wechsler para Adultos - WAIS III, versão adaptada por Nascimento (2005) e Teste Wisconsin de Classificação de Cartas, versão adaptada por Cunha et al. (2005), apresentados no manual do instrumento (Fonseca et al., 2009). Foram também obtidas evidências de fidedignidade a partir das análises de teste-reteste e de concordância entre avaliadores na pontuação de escores em que a subjetividade do avaliador pode afetar os resultados (Fonseca et al., 2009; Pawlowski, 2007).

Além dos métodos já aplicados, também é possível investigar as evidências de validade de construto do instrumento mediante análises fatoriais (Pawlowski, 
Trentini, \& Bandeira, 2007). As análises fatoriais podem ser uma ferramenta adicional para extrair alguns elementos constitutivos do funcionamento cognitivo. Algumas vezes, um fator na análise fatorial pode referirse a um sistema funcional e em outras ocasiões, a elementos da cognição (Ardila \& Bernal, 2007). Segundo descrições mais atuais dos Padrões de Testagem Educacional e Psicológica e a organização encontrada em Urbina (2004), as análises fatoriais são uma fonte de evidência de validade de construto com base na exploração de padrões de convergência e divergência.

Para investigar a validade de construto de um novo instrumento de medida mediante análise fatorial podem ser aplicadas a Análise Fatorial Exploratória (AFE) e a Análise Fatorial Confirmatória (AFC) (Martínez-Arias, Hernández-Lloreda, \& Hernández-Lloreda, 2006). A AFE é amplamente utilizada para avaliar a validade de construto de instrumentos que envolvem medidas de habilidades cognitivas (Carroll, 1993). A aplicação da AFC tem crescido em pesquisas que envolvem a avaliação de modelos cognitivos ou o exame de fatores formados a partir de tarefas e testes cognitivos (Burton, Ryan, Axelrod, \& Schellenberger, 2002; Cockshott, Marsh, \& Hine, 2006; DeJong \& Donders, 2009; Donders, 2008; Wiebe, Espy, \& Charak, 2008).

A AFC é utilizada quando o investigador possui hipóteses prévias sobre o número de fatores e o peso das variáveis em cada um deles, sendo fortemente derivada da teoria e de evidências de pesquisas anteriores. Comparada à AFE, a AFC apresenta, dentre algumas vantagens, a habilidade de especificar a natureza da relação entre os erros de medida (variâncias únicas) dos indicadores, de estimar as relações entre variáveis ajustando erros de medida e de oferecer uma estrutura analítica forte para avaliar a equivalência de modelos de medida entre grupos distintos, por exemplo, com características demográficas diferentes (Brown, 2006). Para a viabilidade de aplicação de análises fatoriais, critérios relacionados aos itens dos instrumentos devem ser atendidos, por exemplo, é recomendada a definição prévia de pelo menos três variáveis para cada possível dimensão do instrumento (Brown, 2006; Fabrigar, Wegener, MacCallum, \& Strahan, 1999).
Dado o exposto, o presente estudo buscou evidências adicionais de validade de construto do Instrumento de Avaliação Neuropsicológica Breve NEUPSILIN mediante a análise fatorial confirmatória. Foram comparados modelos teórico-hipotéticos com modelos alternativos das habilidades cognitivas avaliadas pelo NEUPSILIN.

\section{Método}

\section{Participantes}

A amostra consistiu de 1017 brasileiros, de língua materna portuguesa, residentes na região sul do Brasil, $67.1 \%$ do sexo feminino, com idades de 12 a 90 anos e escolaridade $(M=36.72, D P=23.90)$ de 1 a 27 anos de estudo formal $(M=8.71, D P=4.13)$. Os participantes foram recrutados em ambientes educacionais, empresariais, hospitalares, centros de convivência, entre outros, segundo amostragem por conveniência. Foram critérios de inclusão a ausência de: indícios de lesão cerebral, doença cerebrovascular, epilepsia, doença de Parkinson, hospitalização psiquiátrica e demais condições neurológicas ou psiquiátricas, critérios que foram examinados mediante autorrelato, questionário e instrumentos de triagem. A amostra inicial era composta por 1102 participantes, dos quais 85 foram excluídos por ausência de respostas em algumas tarefas do instrumento ou porque apresentavam uso recente de drogas psicoativas (drogas ilícitas e benzodiazepínicos), dependência ao álcool, indícios de sinais demenciais e presença de depressão em níveis moderado e grave, critérios que foram verificados mediante a aplicação de instrumentos descritos na sessão a seguir do presente estudo.

\section{Instrumentos}

Para a composição da amostra segundo critérios de inclusão e exclusão, foram utilizados: 1) Questionário de dados sócio-demográficos e de condições de saúde: avaliou dados sociais, demográficos eindícios de patologias neurológicas e psiquiátricas; 2) Questionário CAGE (Ewing \& Rouse, 1970), versão de Amaral \& Malbergier (2004), com pontuações de 0 a 4, em que menos de 2 corresponde à negativo para problemas relacionados ao uso de álcool; 3) Mini Exame do Estado Mental - Mini- 
Mental (Folstein, Folstein, \& McHugh, 1975), adaptação de Chaves e Izquierdo (1992): consiste em uma avaliação breve do estado cognitivo, utilizada em triagens para verificação de processos demenciais, cujo escore varia de 0 a 30, sendo ponto de corte 24 para pessoas com 5 anos ou mais de escolaridade e 17 para pessoas com até 4 anos de escolaridade; 4) Inventário Beck de Depressão - BDI (Beck \& Steer, 1993), adaptação e validação de Cunha (2001): permite a classificação de quatro níveis de depressão: mínimo (6-9), leve (10-16), moderado (1729) e grave (30-63), cuja aplicação destinou-se à detecção de estados depressivos em pacientes não idosos e/ou 5) Escala de Depressão Geriátrica - GDS-30 (Yesavage et al., 1983): utilizado como breve triagem de detecção de quadros depressivos na população idosa, com ponto de corte 10 (ausência de depressão).
Como instrumento foco da investigação, foi aplicado o Instrumento de Avaliação Neuropsicológica Breve NEUPSILIN. As evidências de validade de construto deste instrumento foram indicadas por diferenças nas pontuações no NEUPSILIN devido a variável anos de estudo (Pawlowski et al., 2008) e também foram obtidas pela correlação dos escores do instrumento com outros testes que avaliavam construtos similares, por exemplo atenção e inteligência (Fonseca et al, 2009). Além disso, evidências de fidedignidade foram obtidas com análise de teste-reteste, com um índice de confiabilidade variando 0.35 a 0.90 (Fonseca et al., 2009). A organização dos subtestes e diferentes componentes cognitivos avaliados mediante a aplicação das 32 tarefas é demonstrada na Figura 1.

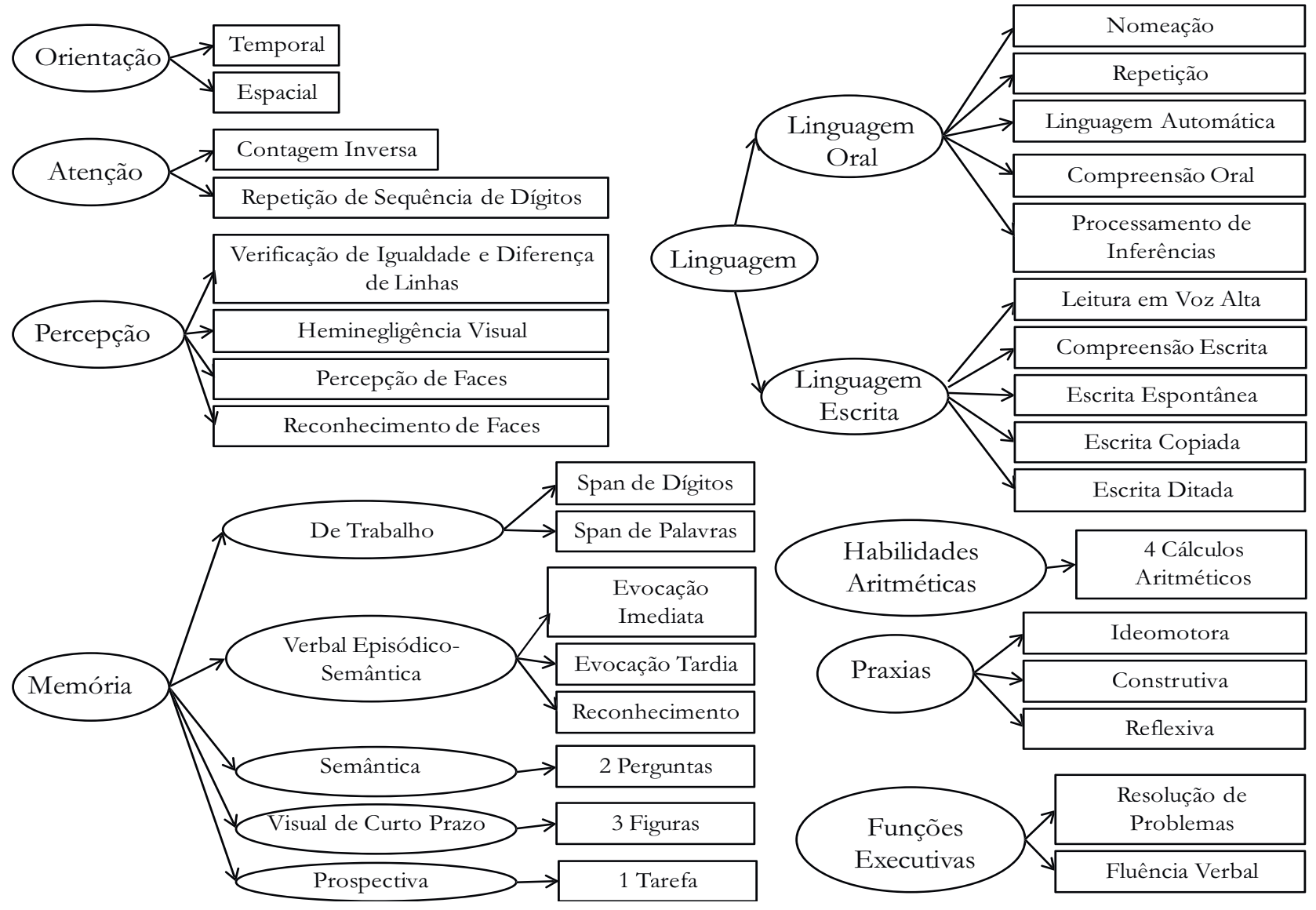

Figura 1. Organização de funções, componentes e tarefas cognitivas avaliadas 


\section{Procedimentos}

A presente investigação constitui-se em um estudo observacional analítico. O projeto foi aprovado pelo Comitê de Ética em Pesquisa da Universidade Federal do Rio Grande do Sul, sob o protocolo de número 2006530 e a coleta foi realizada de acordo com os princípios éticos de pesquisas com seres humanos. Os participantes foram selecionados mediante amostragem por conveniência de população geral. Após triagem de critérios de inclusão, foram explicados os objetivos da pesquisa aos voluntários, que assinaram um Termo de Consentimento Livre e Esclarecido e responderam aos instrumentos de avaliação, conforme a seguinte ordem: questionários, NEUPSILIN, Mini-Mental, BDI ou GDS. A coleta foi individual e realizada por uma equipe de profissionais (psicólogos e fonoaudiólogos) e estudantes de Psicologia devidamente treinados. As aplicações foram realizadas em geral em duas sessões, sendo que o número foi ultrapassado conforme a necessidade ou disponibilidade dos participantes.

\section{Análise dos dados}

Foram realizadas análises descritivas para fins de seleção das variáveis possíveis de inclusão nas análises fatoriais confirmatórias. Para a elaboração dos modelos das diferentes funções cognitivas, considerou-se a estrutura proposta pelas autoras mediante validade de conteúdo do instrumento e foram elaborados modelos alternativos mediante realocação de variáveis ou tarefas do NEUPSILIN que poderiam avaliar mais de um componente cognitivo. Por exemplo, a tarefa de Repetição de Sequência de Dígitos mede atenção, em especial atenção sustentada, e também memória de trabalho. Foram analisados apenas modelos que incluíam no mínimo duas variáveis observáveis para cada variável latente, que apresentavam relação entre no mínimo duas variáveis latentes e que avaliavam construtos de modalidade semelhante, por exemplo, apenas de memória verbal, já que a inserção de variáveis de outra modalidade, por exemplo, visual, poderia comprometer a análise se agrupada em um mesmo modelo.

Foram analisados modelos teórico-hipotéticos e alternativos de linguagem, memória, praxias e funções executivas, compostos pelas variáveis ou tarefas do instrumento. Aplicou-se o método de Mínimos Quadrados Generalizados (Generalized Least Squares) do programa estatístico AMOS (versão 18.0) e foram utilizados os critérios recomendados por Schreiber, Nora, Stage, Barlow e King (2006) para avaliar o índice de qualidade de ajuste dos modelos investigados.

\section{Resultados}

Os resultados de médias e desvios-padrão para os subtestes do NEUPSILIN são apresentados na Tabela 1. Os resultados de indicadores de ajuste das AFCs e os diferentes modelos teórico-hipotéticos e modelos ajustados das funções cognitivas Linguagem, Memória e Praxias encontram-se na Tabela 2 e na Figura 2. Para amostras grandes, em caso de $\chi^{2}$ significativo, utilizase fórmula corrigida $\chi^{2} /$ gl. De acordo com Schreiber et al. (2006), valores de p-exact fit $<0.05, \chi^{2} / \mathrm{gl}<3$, RMSEA $<.05$, p-close fit $>.05$, CFI e GFI $>.95$ são indicativos de adequação de ajuste. Conforme a Tabela 2, apresentaram melhores indicadores de ajuste os modelos de Linguagem Ajustado (A2), Memória Tarefas Verbais (B1) e Praxias Ajustado (C2). Dentre esses, o modelo original de Memória para tarefas verbais, seguindo o modelo teórico-hipotético, foi o que apresentou melhores indicadores de ajuste.

Com base em aspectos teóricos, foram elaborados modelos alternativos para a avaliação de Funções Executivas. Conforme pode ser observado na Figura 3, para fins de análise, agregaram-se outras tarefas do NEUPSILIN ao modelo teórico-hipotético de Funções Executivas proposto pelas autoras, o qual originalmente era composto por apenas duas tarefas. Os modelos 1 e 2 são unidimensionais e incluem as tarefas de Memória de Trabalho, Atenção Sustentada, Resolução de Problemas e Fluência Verbal do NEUPSILIN, com ajuste de erro para o modelo 2 . Os modelos 3 e 4 são de três fatores correlacionados, incluindo correlação para ajuste de erro de medida no modelo 4. Os resultados de indicadores de qualidade de ajuste das AFCs encontram-se na Tabela 3, considerando-se valores de p-exact fit $<.05, \chi^{2} /$ gl $<3$, RMSEA $<.05$, p-close fit $>.05$, CFI e GFI $>.95$ indicativos de adequação 
Tabela 1

Médias e Desvios-padrão para os Subtestes do NEUPSILIN

\begin{tabular}{lcc}
\hline \multicolumn{1}{c}{ Tarefas } & $M$ & $D P$ \\
\hline Orientação Temporal & 3.83 & 0.44 \\
Orientação Espacial & 3.97 & 0.18 \\
Contagem Inversa & 18.69 & 3.92 \\
Repetição Sequência de Dígitos & 3.43 & 2.02 \\
Verificação de Linhas & 5.28 & 0.99 \\
Heminegligência Visual & 1.00 & 0.00 \\
Percepção de Faces & 2.41 & 0.72 \\
Reconhecimento de Faces & 1.89 & 0.33 \\
Ordenamento de Dígitos & 6.98 & 2.23 \\
Span de Palavras em Sentenças & 14.91 & 5.77 \\
Evocação Imediata (MV) & 4.88 & 1.54 \\
Evocação Tardia (MV) & 2.77 & 2.19 \\
Reconhecimento (MV) & 12.97 & 2.51 \\
Memória Semântica de Longo Prazo & 4.63 & 0.59 \\
Memória Visual de Curto Prazo & 2.77 & 0.51 \\
Memória Prospectiva & 1.55 & 0.7 \\
Habilidades Aritméticas & 6.91 & 1.83 \\
Linguagem Oral Nomeação & 4.00 & 0.06 \\
Linguagem Oral Repetição & 9.82 & 0.53 \\
Linguagem Oral Automática & 1.93 & 0.27 \\
Linguagem Oral Compreensão & 2.89 & 0.36 \\
Processamento de Inferências & 2.47 & 0.66 \\
Leitura em Voz Alta (LE) & 11.56 & 1.12 \\
Compreensão Escrita (LE) & 2.85 & 0.41 \\
Escrita Espontânea (LE) & 1.71 & 0.57 \\
Escrita Copiada (LE) & 1.85 & 0.39 \\
Escrita Ditada (LE) & 2.93 & 1.76 \\
Praxia Ideomotora & & 0.15 \\
Praxia Construtiva & 2.35 \\
Praxia Reflexiva & 0.98 \\
Resolução de Problemas & & \\
Fluência Verbal & & \\
\hline Nota. & & \\
\hline
\end{tabular}

Nota MV = Memória Verbal e LE = Linguagem Escrita 
Tabela 2

Indicadores de Qualidade de Ajuste das Análises Fatoriais Confirmatórias para os Modelos Teórico-bipotéticos, Ajustados e Alternativo (N = 1017)

\begin{tabular}{lccccccccc}
\hline \multicolumn{1}{c}{ Modelos } & \multicolumn{7}{c}{ Índice de Qualidade de Ajuste } \\
\cline { 2 - 9 } & $\chi^{2}$ & gl & p-exact fit & $\chi^{2} /$ gl & RMSEA & p-close fit & CFI & GFI \\
\hline A1. Linguagem Oral e Escrita & 112.55 & 34 & $.001 *$ & 3.31 & .048 & .635 & .824 & .978 \\
A2. Linguagem Ajustado & 94.19 & 33 & $.001 *$ & 2.85 & .043 & .874 & .863 & .981 \\
B1. Memória (Tarefas Verbais) & 8.21 & 11 & .694 & 0.75 & .001 & 1.000 & 1.000 & .998 \\
B2. Memória Alternativo & 13.33 & 17 & .714 & 0.78 & .001 & 1.000 & 1.000 & .997 \\
C1. Praxias (Três Tipos) & 72.23 & 32 & .001 & 2.25 & .035 & .989 & .938 & .986 \\
C2. Praxias Ajustado & 17.91 & 12 & .118 & 1.49 & .022 & .992 & .976 & .995 \\
\hline
\end{tabular}

Nota. RMSEA = Root Mean Square Error of Approximation (Raiz da média quadrática do erro de aproximação); CFI = Comparative Fix Index (Índice de Ajuste Comparativo); GFI = Goodness-of-fit Index (Índice de Bondade de Ajuste); * Para amostras grandes, em caso de $\chi^{2}$ significativo, utiliza-se fórmula corrigida $\chi^{2} /$ gl. Valores de p-exact fit $<.05, \chi^{2} / \mathrm{gl}<3$, RMSEA < .05, p-close fit > .05, CFI e GFI > .95 são indicativos de adequação de ajuste (Schreiber et al., 2006).

de ajuste, segundo Schreiber et al. (2006). Conforme a Tabela 3, apresentou melhores indicadores de ajuste o modelo 4, composto por três fatores relacionados: 1) Memória de Trabalho, 2) Atenção Sustentada e 3) Resolução de Problemas e Inibição, respeitando a organização original de tarefas proposta pelas autoras do instrumento para as habilidades de Memória de Trabalho e Atenção Sustentada.

\section{Discussão}

O processo de validação de um novo instrumento de medida depende do número de evidências empíricas que se obtenha mediante a aplicação de métodos de análise e técnicas estatísticas diversas. Para a busca de evidências de validade do NEUPSILIN, diferentes análises foram realizadas, sendo encontrados, em estudos prévios, adequada validade de conteúdo e indicativos de validade de construto (Fonseca et al, 2009; Pawlowski et al, 2008). No presente estudo, buscou-se avaliar novas evidências de validade mediante a aplicação de análises fatoriais confirmatórias para avaliação dos agrupamentos teóricos propostos.

No que se refere aos modelos de linguagem, segundo indicadores de qualidade de ajuste, a AFC indicou melhor adequação para o modelo em que foi realizado ajuste estatístico de erros de medida. Conforme apontado nos resultados dos índices de modificação de ajuste sugeridos pelo programa estatístico, estabeleceu-se a correlação entre os erros das medidas de Compreensão Oral e Compreensão Escrita, o que é esperado teoricamente, visto que são tarefas com instruções e tipos de itens semelhantes. A obtenção de melhor ajuste do modelo, mediante o estabelecimento dessa correlação, confirma que estas duas tarefas compartem um aspecto teórico que não havia sido representado no modelo inicial. Essa análise indicou que os dados empíricos ajustaram-se ao modelo teórico-hipotético para avaliação de linguagem oral e escrita.

Para a análise da função de memória em um modelo teórico, considerou-se a possibilidade de agrupamento de tarefas de memória de categoria verbal. Segundo Fonseca et al. (2008), fazem parte de memória verbal as tarefas do NEUPSILIN que avaliam memória episódica, semântica, prospectiva e de trabalho. Contudo, para a AFC, não foi possível a inclusão da tarefa de Memória Prospectiva no modelo empírico por ela ser mensurada apenas por uma variável observada. Assim, o modelo empírico composto pelas tarefas de avaliação de Memória Verbal Episódico-Semântica (Imediata, Tardia e de Reconhecimento), Memória Semântica de Longo Prazo (avaliando-se as duas perguntas incluídas no instrumento) e de Memória de Trabalho (Span de Dígitos e Span de Palavras em Sentenças) mostrou 


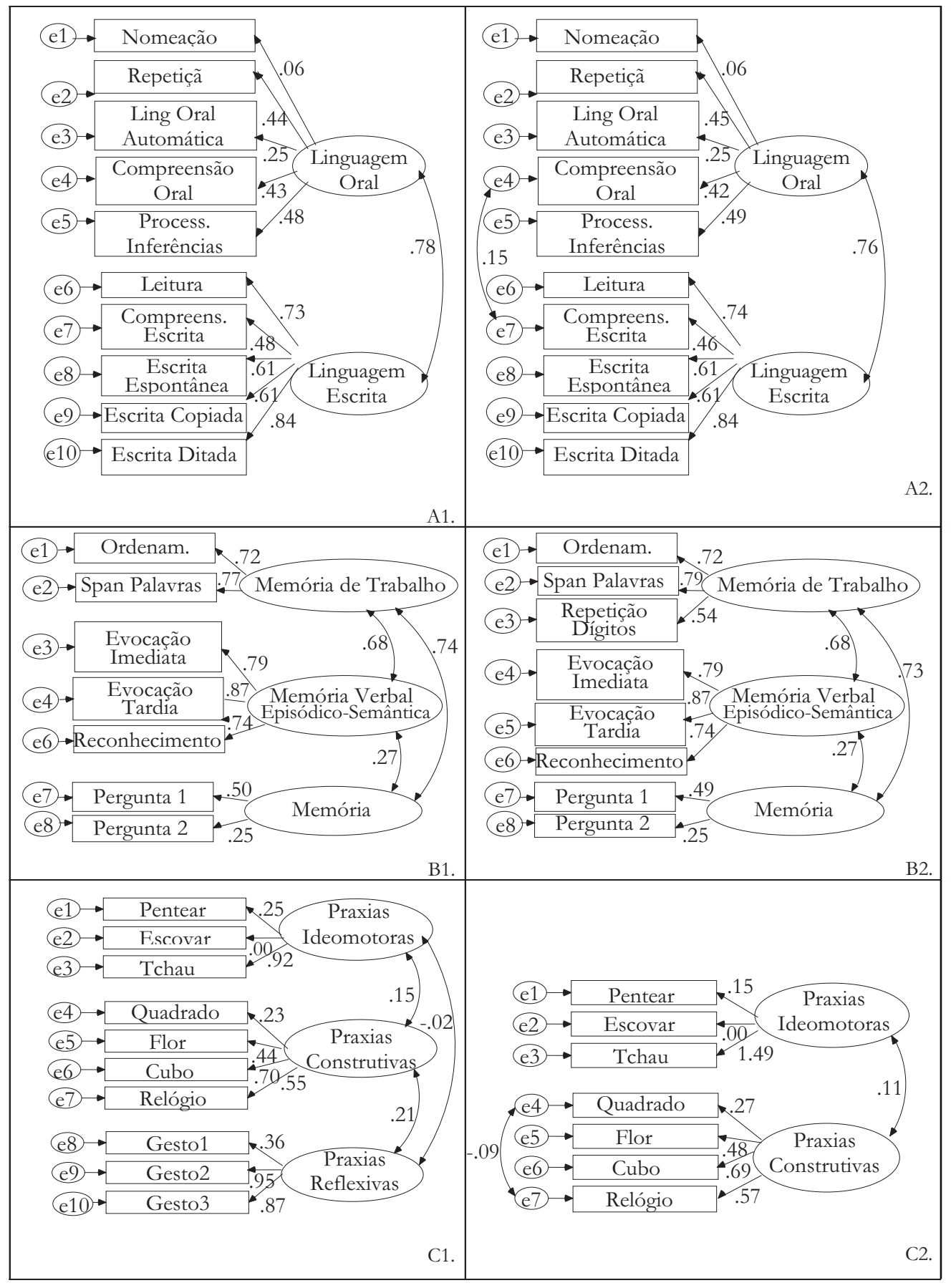

Figura 2. Modelos Teórico-Hipotéticos e Ajustados de Linguagem, Memória e Praxias 


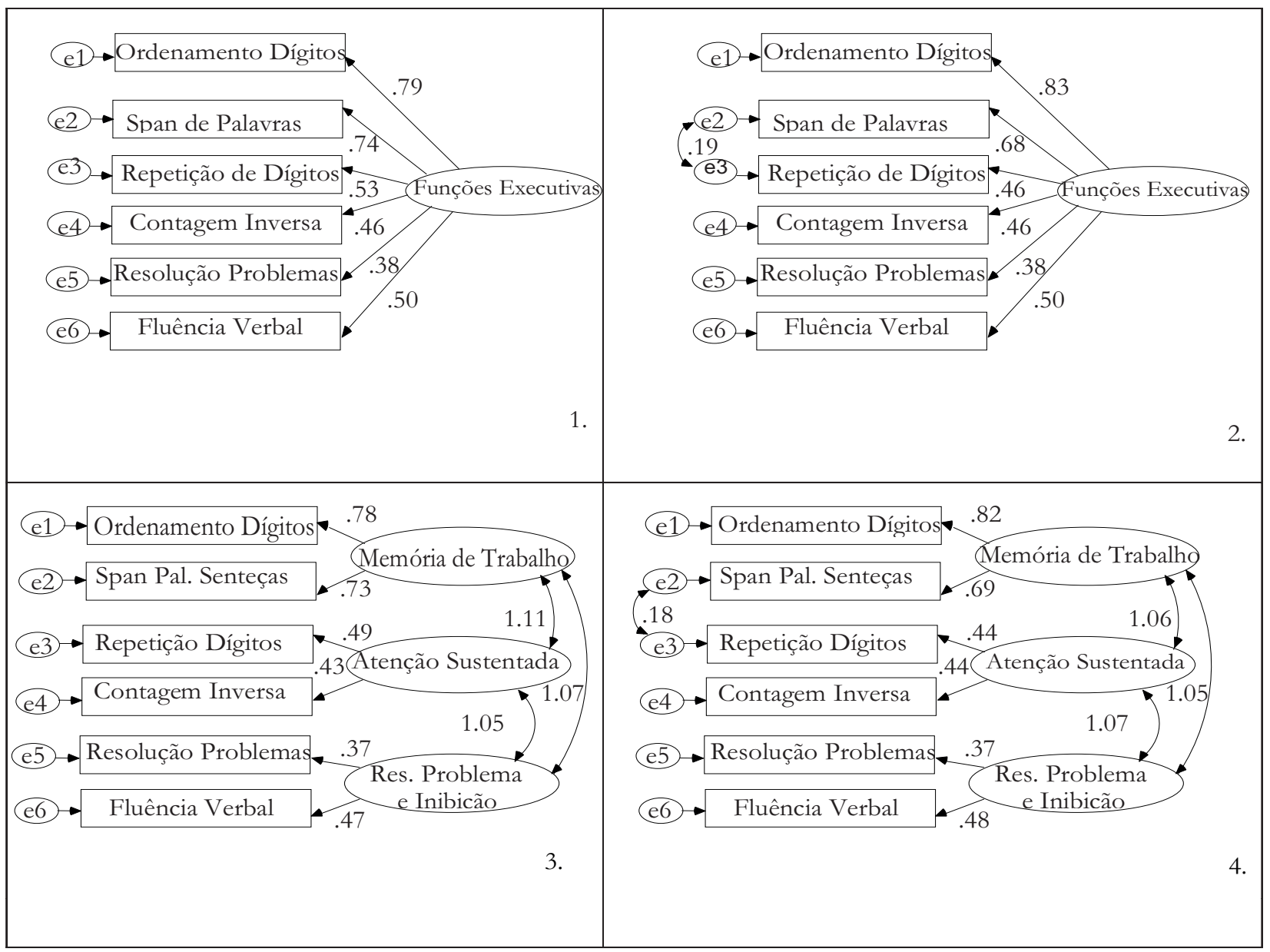

Figura 3. Modelos Alternativos para Avaliação de Funções Executivas

Tabela 3

Indicadores de Qualidade de Ajuste das Análises Fatoriais Confirmatórias para os Modelos Alternativos de Funcão Executiva com Tarefas do NEUPSILIN (N = 1017)

\begin{tabular}{lcccccccc}
\hline \multirow{2}{*}{ Modelo } & \multicolumn{8}{c}{ Índice de Qualidade de Ajuste } \\
\cline { 2 - 9 } & $\chi^{2}$ & $\mathrm{gl}$ & $\mathrm{p}$-exact fit & $\chi^{2} / \mathrm{gl}$ & RMSEA & $\mathrm{p}$-close fit & CFI & GFI \\
\hline 1. Um fator & 25.32 & 9 & .003 & 2.81 & .042 & .715 & .950 & .992 \\
2. Um fator ajustado & 5.32 & 8 & .722 & 0.66 & .001 & .999 & 1.000 & .998 \\
3. Três fatores & 19.14 & 6 & .004 & 3.19 & .046 & .555 & .960 & .994 \\
4. Três fatores ajustado & 3.97 & 5 & .554 & 0.79 & .001 & .990 & 1.000 & .999 \\
\hline
\end{tabular}

Nota. Valores de p-exact fit $>.05, \chi^{2} / \mathrm{gl}<3$, RMSEA $<.05$, p-close fit $>.05$, CFI e GFI $>.95$ são indicativos de adequação de ajuste (Schreiber et al., 2006). 
ajuste adequado, fornecendo apoio empírico ao modelo teórico-hipotético para avaliação de memória de categoria verbal. Quando foram inseridos no modelo tarefas de outra categoria, os índices de adequação dos ajustes estatísticos mostram-se piores, não sendo inclú́dos esses modelos neste estudo. Já o modelo alternativo que inclui a tarefa de Repetição de Sequência de Dígitos no modelo de memória verbal também indicou adequados índices de ajuste. Confirma-se, por resultados estatísticos, que a tarefa de Repetição de Sequência de Dígitos inclui componentes de avaliação do construto de memória de trabalho.

No que se refere à avaliação de praxias, o modelo empírico ajustado incluindo as tarefas de Praxias Idemotora e Construtiva apresentou índices melhores que o modelo original em razão de as três respostas de Praxia Reflexiva referirem-se à avaliação de uma seqüência de gestos, o que possivelmente ocasionou a redução de variabilidade de respostas quando separadas em três alternativas. Portanto, o modelo empírico indicou a adequação de praxias construtiva e ideomotora, mas a confirmação da adequação em termos estatísticos de tarefas de praxias do tipo reflexiva requer a inclusão de um maior número de tarefas ou outro método de análise.

Considerando a teoria de avaliação de funções executivas que integra a avaliação de memória de trabalho, atenção sustentada, resolução de problemas e inibição, entre outras habilidades, como planejamento, formação conceitual, desenvolvimento estratégico e implementação (Ardila, 2008; Chan, Shum, Toulopoulou, \& Chen, 2008; Hull, Martin, Beier, Lane, \& Hamilton, 2008; Salthouse, 2005), buscou-se avaliar modelos alternativos de funções executivas formadas a partir de tarefas incluídas no NEUPSILIN. Os modelos foram formados com as tarefas de Atenção Sustentada, Memória de Trabalho, Resolução de Problemas e Fluência Verbal.

Entre os quatro modelos avaliados, o modelo que segue a organização proposta teoricamente apresentou melhores índices de ajuste. Este modelo inclui a avaliação de três dimensões: 1) Memória de Trabalho, incluindo as tarefas Ordenamento Ascendente de Dígitos e Span de Palavras em Sentenças, 2) Atenção, abrangendo as tarefas de Repetição de Sequência de Dígitos e Contagem Inversa, e 3) Resolução de Problemas e Inibição, na qual foram inseridas as tarefas de Resolução de Problemas e Fluência Verbal, as quais pertencem à dimensão Função Executiva no modelo teórico original proposto pelas autoras do instrumento NEUPSILIN.

Para o ajuste deste modelo, foi necessário estabelecer a correlação dos erros de medida das tarefas Repetição de Sequência de Dígitos e Span de Palavras em Sentenças. A correlação de erros de medida, utilizando sugestões do programa estatístico para índices de modificação de ajuste, aponta que estas duas tarefas compartem algum aspecto teórico que não foi representado no modelo. Hipotetizase que um fator latente, que pode ser a memória de curto prazo, integre as habilidades requeridas para a realização das tarefas Repetição de Sequência de Dígitos do fator de Atenção Sustentada e Span de Palavras em Sentenças do fator Memória de Trabalho.

Ressalta-se que os componentes de atenção e memória frequentemente estão relacionados um ao outro e a outras habilidades cognitivas, por exemplo, às funções executivas, relações que ainda não foram completamente compreendidas (Ostrosky-Solís et al., 2007). Por exemplo, no que se refere à estrutura estatística do componente executivo central da memória de trabalho, Miyake et al. (2000) avaliou as funções flexibilidade mental, atualização da informação e inibição da resposta predominante e concluiu que ao mesmo tempo que essas funções podem ser evidentemente distinguidas, elas também dividem algumas características. Além disso, os autores sugerem que a habilidade de coordenar duas atividades simultaneamente é independente das outras três funções executivas que eles estudaram.

No que se refere ao agrupamento proposto no modelo de funções executivas deste estudo, evidenciou-se a adequação da medida de Atenção incluindo as duas tarefas conforme modelo original. Para estudos futuros, sugerese a avaliação de um modelo que possua mais tarefas de resolução de problemas e inibição a fim de ser possível a separação destas duas tarefas em duas dimensões, objetivando-se confirmar a adequação do modelo proposto neste estudo para avaliar Função Executiva. 
Em conclusão, as análises realizadas apóiam os resultados obtidos previamente de validade de conteúdo do instrumento e reforçam, mediante validade de construto, sua adequação para medir as funções de linguagem, memória de categoria verbal, praxias e funções executivas. Apesar de limitações de análise no presente estudo, entende-se que as tarefas das funções de Orientação e Habilidades Aritméticas constituemse medidas adequadas teoricamente, em razão da confirmação de seu uso em outros instrumentos, por exemplo, no Mini-Mental.

Como limitação dos resultados descritos neste estudo, observou-se que as tarefas de Percepção, Memória Visual de Curto Prazo e Memória Prospectiva necessitam ser avaliadas mediante outros delineamentos ou técnicas de análise que não foram contemplados nesse estudo. Além disso, a decisão de uma amostra saudável com diferentes níveis de idade e escolaridade como fonte de validação do instrumento incluiu a presença de casos com efeito de teto em algumas tarefas e pode ter contribuído para a pouca variabilidade dos escores, o que resultou na impossibilidade de avaliação de algumas variáveis do instrumento. É importante considerar o tipo de amostra ou população em que as análises fatoriais são conduzidas, pois podem limitar a generalização dos resultados para outras populações, em especial quando se analisa populações com diferentes doenças (Greenaway, Smith, Tangalos, Geda, \& Ivnik, 2009). Portanto, sugere-se replicar as análises fatoriais confirmatórias realizadas neste estudo com amostras clínicas.

Também sugere-se a realização, em outros estudos com amostras mais heterogêneas ou clínicas, de análises de invariância dos modelos para sexo e grupos de idade. Ainda não foram apresentadas evidências de que o instrumento NEUPSILIN seja sensível para gênero e as análises de invariância para grupos de idade não foram viáveis com a amostra do presente estudo em razão da variância dos dados com amostra sem danos neurológicos ter sido insuficiente para tais análises. Em relação a isso, Park et al. (2012) avaliaram a estrutura fatorial de uma bateria neuropsicológica entre adultos idosos com diferentes níveis de severidade de demência e encontraram cinco fatores que foram invariantes para níveis de prejuízo cognitivo. Dessa forma, propõemse para estudos futuros que se realizem análises de invariância de modelos para diferentes níveis de comprometimento cognitivo, analisando-se amostras de grupos clínicos, as quais podem gerar dados com maior variância, o que permite a execução da análise fatorial. Faz-se necessário, portanto, realizar novos estudos com delineamentos diferentes objetivando a confirmação das evidências de validade do Instrumento de Avaliação Neuropsicológica Breve NEUPSILIN.

\section{Referências}

American Educational Research Association, American Psychological Association, \& National Council of Measurement in Education. (1999). Standardsfor educational and psychological testing. Washington, DC: AERA.

Ardila, A. (2008). On the evolutionary origins of executive functions. Brain and Cognition, 68, 92-99.

Ardila, A., \& Bernal, B. (2007). What can be localized in the brain? Toward a "factor" theory on brain organization of cognition. International Journal of Neuroscience, 117, 935-937.

Amaral, R. A., \& Malbergier, A. (2004). Avaliação do instrumento de detecção de problemas relacionados ao álcool (CAGE) entre trabalhadores da prefeitura dos Campi da Universidade de São Paulo (USP) - Campus Capital. Revista Brasileira de Psiquiatria, 26(3), 156-183.

Beck, A. T., \& Steer, R. A. (1993). Manual for the Beck Depression Inventory. San Antonio, TX: Psychological Corporation.

Brown, T. A. (2006). Confirmatory Factor Analysis for Applied Research. New York: The Guilford Press.

Burton, B., Ryan, J. J., Axelrod, B. N., \& Schellenberger, T. (2002). A confirmatory factor analysis of the WAIS-III in a clinical sample with crossvalidation in the standardization simple. Archives of Clinical Neuropsychology, 17, 371-387.

Cambraia, S. V. (2002). AC - Teste de atenção concentrada. São Paulo: Vetor. 
Campos, F. (2003). Teste das Matrizes Progressivas - Escala Geral. Rio de Janeiro: CEPA.

Carroll, J. B. (1993). Human Cognitive Abilities: A Survey of Factor Analytic Studies. New York: Cambridge University Press.

Chaves, M. L. \& Izquierdo, I. (1992). Differential diagnosis between dementia and depression: a study of efficiency increment. Acta Neurologica Scandinavia, 11, 412-429.

Chan, R. C. K., Shum, D., Toulopoulou, T., \& Chen, E. Y. H. (2008). Assessment of executive functions: Review of instruments and identification of critical issues. Archives of Clinical Neuropsychology, 23, 201-216.

Cockshott, F. C., Marsh, N. V., \& Hine, D. W. (2006). Confirmatory Factor Analysis of the Wechsler Intelligence Scale for Children - Third Edition in an Australian Clinical Sample. Psychological Assessment, 18(3), 353-357.

Cunha, J. A. (2001). Escalas Beck. São Paulo: Casa do Psicólogo.

Cunha, J. A., Trentini, C. M., Argimon, I., Oliveira, M. S., Werlang, B. G., \& Prieb, R. G. G. (2005). Manual do Teste Wisconsin de Classificação de Cartas. São Paulo, SP: Casa do Psicólogo.

Donders, J. (2008). A confirmatory factor analysis of the California Verbal Learning Test - Second Edition (CVLT-II) in the standardization sample. Assessment, 15(2), 123-131.

DeJong, J., \& Donders, J. (2009). A confirmatory factor analysis of the California Verbal Learning Test - Second Edition (CVLT-II) in a traumatic brain injury sample. Assessment, 16(4), 328-336.

Ewing, J. A., \& Rouse, B. A. (1970). Identifying the bidden alcoholic. In Program and abstracts of the 29th International Congress on Alcohol and Drug Dependence. Sidney, Australia.

Fabrigar, L. R., Wegener, D. T., MacCallum, R. C., \& Strahan, E. J. (1999). Evaluating the use of exploratory factor analysis in psychological research. Psychological Methods, 4(3), 272-299.
Fagerström, K. O., \& Schneider, N. G. (1989). Measuring nicotine dependence: A review of the Fagerström Tolerance Questionnaire. Journal of Behavioral Medicine, 12(2), 159-182.

Folstein, M. F., Folstein, S. E., \& McHugh, P. R. (1975). Mini-mental state. Journal of Psychiatry Resources, 12, 189-198.

Fonseca, R. P., Salles, J. F. \& Parente, M. A. M. P. (2009). Instrumento de Avaliação Neuropsicológica Breve NEUPSILIN. São Paulo: Vetor.

Fonseca, R. P., Salles, J. F., \& Parente, M. A. M. P. (2008). Development and content validity of the Brazilian Brief Neuropsychological Assessment Battery NEUPSILIN. Psychology \& Neuroscience, 1(1), 55-62.

Greenaway, M. C., Smith, G. E., Tangalos, E. G., Geda, Y. E., \& Ivnik, R. J. (2009). Mayo Older Americans Normative Studies: Factor Analysis of an Expanded Neuropsychological Battery. The Clinical Neuropsychologist, 23(1), 7-10.

Hull, R., Martin, R. C., Beier, M. E., Lane, D., \& Hamilton, A. C. (2008). Executive function in older adults: A structural equation modeling approach. Neuropsychology, 22(4), 508-522.

Lezak, M. D., Howieson, D. B., \& Loring, D. W. (2004). Neuropsychological Assessment. New York: Oxford University Press.

Marques, A. C. P. R., Campana, A., Gigliotti, A. P., Lourenço, M. T. C., Ferreira, M. P., \& Laranjeira R. (2001). Consenso sobre o tratamento da dependência de nicotina. Revista Brasileira de Psiquiatria, 23(4), 200-214.

Martínez-Arias, M. R., Hernández-Lloreda, M. J., \& Hernández-Lloreda, M. V. (2006). Psicometría. Madrid: Alianza Editorial.

Miyake, A., Friedman, N. P., Emerson, M. J., Witzki, A. H., Howerter, A., \& Wager, T. D. (2000). The unity and diversity of executive functions and their contributions to complex "Frontal Lobe" tasks: a latent variable analysis. Cognitive Psychology, 41, 49-100.. 
Nascimento, E. (2005). WAIS-III: Escala de Inteligência Wechsler para Adultos: Manual técnico. São Paulo: Casa do Psicólogo.

Ostrosky-Solís, F., Gómez-Pérez, M. E., Matute, E., Rosselli, M., Ardila, A., \& Pineda, D. (2007). NEUROPSI ATTENTION AND MEMORY: A Neuropsychological Test Battery in Spanish with Norms by Age and Educational Level. Applied Neuropsychology, 14(3), 156-170.

Park, L. Q., Gross, A. L., McLaren, D. G., Pa, J., Johnson, J. K., Mitchell, M., \& Manly, J. J. (2012). Confirmatory factor analysis of the ADNI Neuropsychological Battery. Brain Imaging and Behavior, 6(4), 528-539.

Pasquali, L. (2010). Instrumentação Psicológica: Fundamentos e Prática. Porto Alegre: Artmed.

Pawlowski, J. (2007). Evidências de validade e fidedignidade do Instrumento de Avaliação Neuropsicológica Breve NEUPSILIN. Dissertação de Mestrado, Programa de Pós-Graduação em Psicologia, Instituto de Psicologia, Universidade Federal do Rio Grande do Sul. Porto Alegre, RS.

Pawlowski, J., Trentini, C. M., \& Bandeira, D. R. (2007). Discutindo procedimentos psicométricos a partir da análise de um instrumento de avaliação neuropsicológica breve. Psico-USF, 12(2), 211-219.

Pawlowski, J., Fonseca, R. P., Salles, J. F., Parente, M. A. M. P., \& Bandeira D. R. (2008). Evidências de validade do Instrumento de Avaliação Neuropsicológica Breve NEUPSILIN. Arquivos Brasileiros de Psicologia, 60 (2), 101-116.
Raven, J. C., Court, J. H., \& Raven, J. (1992). Standard Progressive Matrices. Manual. Oxford: Oxford Psychologists Press.

Schreiber, J. B., Nora, A., Stage, F. K., Barlow, E. A., \& King, J. (2006). Reporting structural equation modeling and confirmatory factor analysis results: a review. The Journal of Educational Research, 99(6), 323-337.

Salthouse, T. A. (2005). Relations between cognitive abilities and measures of executive functioning. Neuropsychology, 19(4), 532-545.

Strauss, E., Sherman, E. M. S., \& Spreen, O. (2006). A Compendium of Neuropsychological Tests: Administration, Norms and Commentary. New York: Oxford University Press.

Tonglet, E. C. (2003). Bateria Geral de Funções Mentais: Teste de Atenção Concentrada - BGFM-2 (1ª ed.). São Paulo: Vetor Editora.

Urbina, S. (2004). Essentials of Psychological Testing. New Jersey: John Wiley \& Sons, Inc.

Wiebe, S. A., Espy, K. A., \& Charak, D. (2008). Using Confirmatory Factor Analysis to Understand Executive Control in Preschool Children: I. Latent Structure. Development Psychology, 44(2), 575-587.

Yesavage, J. A., Brink, T. L., Rose, T. L., Lum, O., Huang, V., Adey, M., Leirer, V. O. (1983). Development and validation of a geriatric depression screening scale: a preliminary report. Journal of Psychiatric Research, 17, 37-49. 


\section{Apêndice}

Funções e Componentes Avaliados, Descrição e Pontuação Minima e Máxima das Tarefas do NEUPSILIN, conforme Fonseca et al. (2008)

\begin{tabular}{|c|c|c|}
\hline Funções e Componentes Avaliados & Descrição das Tarefas & Pontuação \\
\hline \multicolumn{3}{|l|}{ 1.0 Orientação Têmporo-espacial } \\
\hline 1.1 Tempo & Resposta de dia da semana, dia do mês, mês e ano & $0-4$ \\
\hline 1.2 Espaço & Resposta de local, cidade, estado e país & $0-4$ \\
\hline \multicolumn{3}{|l|}{ 2.0 Atenção Sustentada } \\
\hline 2.1 Contagem Inversa & Contagem de 50 a 30 pelo examinando & $0-20$ \\
\hline 2.2 Repetição de Seqüência de Dígitos & Repetição de uma seqüência de sete números & $0-7$ \\
\hline \multicolumn{3}{|l|}{ 3.0 Percepção } \\
\hline $\begin{array}{l}3.1 \text { Verificação de Igualdade e Diferença de } \\
\text { Linhas }\end{array}$ & $\begin{array}{l}\text { Verificação de extensão de similaridade ou diferença entre } \\
\text { seis pares de linhas }\end{array}$ & $0-6$ \\
\hline 3.2 Heminegligência Visual & $\begin{array}{l}\text { Percepção de todo espaço de uma folha de papel com } \\
\text { traços que devem ser riscados }\end{array}$ & $0-1$ \\
\hline 3.3 Percepção de Faces & $\begin{array}{l}\text { Avaliação, como iguais ou diferentes, de três pares de } \\
\text { fotografias de faces (frente e perfil) }\end{array}$ & $0-3$ \\
\hline 3.4 Reconhecimento de Faces & $\begin{array}{l}\text { Reconhecimento, entre quatro desenhos de faces, de duas } \\
\text { faces inicialmente apresentadas }\end{array}$ & $0-2$ \\
\hline \multicolumn{3}{|l|}{ 4.0 Memória } \\
\hline \multicolumn{3}{|l|}{ 4.1 Memória de Trabalho } \\
\hline 4.1.1 Ordenamento Ascendente de Dígitos & $\begin{array}{l}\text { Repetição, em ordem crescente, de dez conjuntos variando } \\
\text { de dois a seis dígitos }\end{array}$ & $0-10$ \\
\hline $\begin{array}{l}\text { 4.1.2 Span Auditivo de Palavras em } \\
\text { Sentenças }\end{array}$ & $\begin{array}{l}\text { Repetição de sentenças verbais apresentadas pelo } \\
\text { examinador e memorização da palavra final de cada } \\
\text { sentença; ao final, evocação em ordem das últimas palavras } \\
\text { de cada sentença apresentada (conjuntos de duas, três, } \\
\text { quatro e cinco sentenças) }\end{array}$ & $0-28$ \\
\hline \multicolumn{3}{|l|}{ 4.2 Memória Verbal Episódico-Semântica } \\
\hline 4.2.1 Evocação Imediata & Evocação livre de nove palavras ditas pelo examinador & $0-9$ \\
\hline 4.2.2 Evocação Tardia & $\begin{array}{l}\text { Evocação livre das mesmas nove palavras após tarefas de } \\
\text { linguagem oral }\end{array}$ & $0-9$ \\
\hline 4.2.3 Reconhecimento & $\begin{array}{l}\text { Reconhecimento das nove palavras da lista alvo em uma } \\
\text { lista de } 18 \text { palavras ( } 9 \text { palavras-alvo e } 9 \text { distratores) }\end{array}$ & $0-18$ \\
\hline 4.3 Memória Semântica & $\begin{array}{l}\text { Resposta a duas perguntas referentes a conhecimentos } \\
\text { gerais }\end{array}$ & $0-5$ \\
\hline 4.4 Memória Visual de Curto Prazo & $\begin{array}{l}\text { Reconhecimento, em três tarefas, de uma figura sem } \\
\text { sentido entre um conjunto de três estímulos }\end{array}$ & $0-3$ \\
\hline 4.5 Memória Prospectiva & $\begin{array}{l}\text { Execução, ao final da avaliação, da escrita do nome em } \\
\text { uma folha de papel segundo instrução dada no início da } \\
\text { avaliação }\end{array}$ & $0-2$ \\
\hline
\end{tabular}

Continue... 
Funções e Componentes Avaliados, Descrição e Pontuação Mínima e Máxima das Tarefas do NEUPSILIN, conforme Fonseca et al. (2008)

Funções e Componentes Avaliados

Descrição das Tarefas

Pontuação

5.0 Habilidades Aritméticas

Resolução de quatro cálculos, um de cada operação 0 -8 aritmética básica

6.0 Linguagem

6.1 Linguagem Oral

6.1.1 Nomeação

Nomeação de dois objetos e duas figuras

$0-4$

6.1.2 Repetição

6.1.3 Linguagem Automática

Repetição de oito palavras reais e duas pseudopalavras

$0-10$

Contagem de um a dez e evocação de todos os meses do

$0-2$ ano, em ordem

6.1.4 Compreensão Oral

Indicação de figuras correspondentes a palavras e sentenças

$0-3$ ditas pelo examinador (três estímulos)

6.1.5 Processamento de Inferências

\subsection{Linguagem Escrita}

6.2.1 Leitura em Voz Alta

Explicação do significado de um provérbio e de duas metáforas

Leitura em voz alta de dez palavras reais e de duas $0-12$ pseudopalavras

6.2.2 Compreensão Escrita

Indicação de figuras correspondentes ao significado de $0-3$ palavras e frases lidas (três estímulos)

6.2.3 Escrita Espontânea

Escrita de uma sentença

$0-2$

6.2.4 Escrita Copiada

Cópia de uma sentença

$0-2$

6.2.5 Escrita Ditada

Escrita de dez palavras reais e de duas pseudopalavras

7.0 Praxias

7.1 Ideomotora

Execução de três gestos, conforme instrução verbal do

$0-3$ examinador

7.2 Construtiva

Cópia de três figuras (quadrado, flor e cubo) e desenho de

$0-16$

7.3 Reflexiva um relógio

Repetição de uma seqüência de três gestos, de acordo com 0 - 3 um modelo dado

8.0 Funções Executivas

8.1 Resolução de problemas

Resposta a duas questões envolvendo raciocínio abstrato

$0-2$

8.2 Fluência Verbal

Verbalização, durante um minuto, de palavras que iniciem

$0-7$ com a letra $\mathrm{F}$ 